An important section of the work is that on stress corrosion, and research at present in progress on the occurrence of this phenomenon in high-tensile brasses, aluminium-base and magnesium-base alloys is being co-ordinated with the simultaneous objectives of elucidating the behaviour of particular materials under certain conditions and of providing a basic understanding of the mechanism of stress corrosion without which the behaviour of different materials in service can never be adequately understood. Similarly, in the case of the jointing of metals, the researches mentioned above on soldering have provided information of a practical nature on the relative merits of different solders in certain applications, but the basic factors underlying the soldering operations are not understood. The Association's investigation is being deflected into a study of these factors, without knowledge of which further practical advances can only be on a hit-and-miss basis.

Other examples could be quoted, but those described illustrate the type of work which is appropriate to the British Non-Ferrous Metals Research Association and on which it will concentrate more and more in the future as the realization of its expanded programme becomes possible. It is not intended that the Association should abandon the study of practical problems in favour of fundamental ones, but that the two aspects should be combined by carrying work on questions of practical importance to a stage at which the fundamental principles underlying the phenomena observed are fully understood.

\section{CYTOLOGY, BIOPHYSICS AND BIOCHEMISTRY}

$\mathrm{O}^{\mathrm{N}}$ November 10, the Biochemical Society held a discussion meeting under the general title "The Chemical Basis of Cell Structure and Function". The main contributors, in order of speaking, were Dr. J. F. Danielli, Dr. J. H. Schulman, Prof. W. T. Astbury, Dr. E. Stedman, Dr. J. N. Davidson and Prof. E. J. Conway.

Dr. Danielli said that the task of building up a dynamic picture of the cell, even at the chemical level, involves knowledge of the permeability of the plasma membrane, the cytoplasm, the nuclear membrane, the nucleoplasm, and the various other formed bodies and organs of the cell, such as granules, vacuoles and mitochondria. At present we have a great deal of information about the permeabilities of the plasma membrane, and a considerable knowledge of the permeability of the ground substance of cytoplasm, but otherwise there are few facts available. The permeability of the ground substance of cytoplasm is high : the resistance to diffusion within it, for molecules up to the size of Trypan blue, is not much greater than that encountered in water. The permeability of the plasma membrane is often very low : the rates at which different molecules penetrate a given membrane differ enormously, and cells of different types and differing species exhibit very large differences in permeability to the same molecule. However, when the permeability of a cell to, say, three or four different molecular species is known, it is usually possible to calculate the order of magnitude of the permeability to most other molecular species. The mechanism of penetration through the plasma membrane was discussed, and it was pointed out that further advances in this part of the field are held up by the inadequacy of present-day theories of diffusion.

Of certain aspects of plasma membrane permeability we are still almost completely ignorant : these aspects include permeability to large polar molecules, such as proteins, and to large non-polar molecules, such as fats and sterols. As yet practically untouched are all secretory processes- that is, all those activities of the cell involving the movement of molecules by processes other than simple diffusion.

Dr. Schulman discussed lipo-protein associations, as revealed by the study of emulsions and monolayers. If a globular protein is brought into contact with a lipoid at an interface, it will unroll if association takes place. In vitro complete reversal of this unrolling process is very difficult, but the suggestion was put forward that in vivo reversal may be more readily achieved. Dr. Schulman said that in his experiments there is a marked tendency for the adsorption of proteins to be influenced by the sign of charge of the protein and of the lipoid surface. When the surfaces are of opposite sign of charge, vigorous adsorption of protein takes place, whereas when protein and lipoid have the same charge, often no adsorption occurs ; in fact, if a protein on the acid side of its iso-electric point is allowed to become adsorbed on a negatively charged interface, and then the $p \mathrm{H}$ is shifted so as to make both surface and protein of the same sign of charge, desorption of protein will often occur. The desorbed protein may be monodisperse.

When the monolayer technique is used to study protein-lipoid association, it is convenient to spread a monolayer of lipoid, and inject protein into the underlying aqueous phase. If the protein penetrates the lipoid film, this is reflected by changes of surface pressure, surface viscosity and surface potential. In this way it can be shown that cholesterol absorbs many proteins very strongly. Lecithin has not so far been found to absorb any protein over the physiological $p H$ range. Kephalin associates with some proteins at $p \mathrm{H} 3 \cdot 7$. With long-chain amines, association appears to depend in part on the length of the paraffin chain of the amine.

Prof. Astbury pointed out that, although the fine details of protein structure remain to be elucidated, some of the broader outlines are now beginning to appear. Two broad groupings of proteins have appeared in the light of physical studies : the keratinmyosin-fibrinogen (k.m.f.) group and the collagen group. These groupings exist despite marked variation in amino-acid composition within both groups. The k.m.f. group is distinguished by long-range elasticity, based on a particular method of folding of the main polypeptide chains of the protein : it now appears probable that the side chains are closely packed, with polar chains lying on one side and nonpolar chains on the other side of the main chain. The basic plan for synthesis of the k.m.f. group is a standard plan apparently possessed by cells of highly diverse types ; the details of this plan can be adapted to a variety of ends, as the processes of differentiation demand.

The proteins of the collagen group have their polypeptide chains extended in a manner differing from that which occurs in the k.m.f. group. The analysis of this has not proceeded so far as for the k.m.f. group. It is, however, possible that reticulin connective fibres have a structure which is intermediate between that of the two groups. 
The impression given by combining $\mathrm{X}$-ray and electron microscope studies is of successive levels of organisation. For example, with X-rays one of the repetition units is as large as $640 \mathrm{~A}$., and Schmitt and his colleagues, using the electron microscope, have demonstrated a corresponding system of light and dark bands in fine collagen fibres. We must regard fibrous proteins as built up first of amino-acids combined to form larger units, which in turn form part of larger units, step succeeding step until the fibril and combination of fibrils of the microscopist are attained. In the building of these larger structures, small amounts of non-protein components may take an essential role; it is therefore necessary to estimate the proportions of such substances as sugars and nucleotides in proteins with much greater precision than hitherto.

Dr. Stedman said that whereas the nuclei of salmon sperm contain protamine and no histone, the somatic nuclei of salmon contain histone and no protamine : in consequence, neither protamine nor histone can provide the chemical basis of genetic activity. Nucleic acid and the acidic protein chromosomin are the remaining constituents of cell nuclei which must be considered. Nucleic acid, in his opinion, appears to be of invariable composition, and therefore cannot convey genetic activity, but probably forms part of the nuclear sap. But chromosomins, which appear to be a universal constituent of cell nuclei, probably have this function.

Chromosomins, unlike histones, are rich in tryptophane. At present they can only be isolated in the denatured condition. The chromosomins so isolated should be strictly comparable to the characteristic sectioned or smeared material of the cytologist, which also is denatured. It is therefore of great interest that denatured chromosomin has staining properties closely resembling those of chromatin. In Dr. Stedman's opinion the basie proteins (protamines and histones) probably form part of the cell sap, and are of importance in the control of cell proliferation.

Dr. Davidson summarized much of the recent work on cytoplasmic ribonucleoproteins. Strictly, this material should be referred to as pentose nucleoprotein, since the pentose has been identified as ribose only for the polynucleotides of yeast and liver. Brachet first described the ribonuclease test for ribonucleic acid: in this test, tissue sections are treated with purified ribonuclease, which attacks ribonucleic acid but not deoxyribonucleic acid (found almost exclusively in cell nuclei). Material which stains with such basic stains as pyronine and toluidine blue, and is removed by this enzyme action, is taken as ribonucleic acid.

Adult liver cells, when centrifuged, exhibit four strata : the heaviest material is glycogen, the next stratum contains the nucleus and large particles including mitochondria, the third layer chromophilic ground-substance and the lightest layer true hyaloplasm. When disintegrated cells are separated into fractions by differential centrifugation, two main sizes of particle are found containing ribonucleoprotein. The larger $(0 \cdot 5-2 \mu)$ are believed to be the mitochondria and secretory granules, and the smaller microsomes $(50-200 \mathrm{mu})$ to be the chromophilic groundsubstance.

In contrast, in the chick embryo, yeast, amphibian eggs in development and in young oocytes, it is reported that $60-80$ per cent of the ribonucleic acid is not sedimentable in the ultracentrifuge. There is much evidence that cytoplasmic ribonucleic acid is closely connected with metabolism, and the view has been advocated by several authors that cytoplasmic ribonucleoprotein particles are organs responsible for protein synthesis.

Prof. Conway presented an analysis of the distribution of inorganic constituents of cells, with particular reference to muscle cells. The most interesting aspect of this was the recent work from Prof. Conway's own laboratory, in which it has been shown that, subject to certain assumptions, ions distribute them selves across the muscle fibre membrane according to the Gibbs-Donnan equilibrium. The assumptions are that the muscle membrane has pores permeable to hydrogen ions, potassium ions, and small anions, but not to sodium ions and large anions. With these assumptions it follows that, as a cell grows, potassium ions will be automatically accumulated in the cell as phosphate esters, and proteins are synthesized. If these assumptions are justified, we must assume that such ions as calcium and magnesium, being larger than sodium, will also be unable to enter through the membrane pores. But they will be able to form un-ionized complexes with, say, cell membrane lipoids, and then penetrate into the cell in this form.

In the ensuing discussion many points were raised, among the more important of which were that emphasis must be laid on the lack of satisfactory evidence that nucleic acids are chemically homogeneous, and that doubt must be felt as to whether muscle cell membranes are in fact impermeable to sodium ions. Until the constitution of the nucleic acids is more precisely defined, it will presumably be difficult to fix limits to their potentialities in vivo.

With regard to the permeability of muscle fibre membranes to sodium ions, it is difficult to come to any final conclusion without studies with isotopic sodium : the position at present may be construed as involving either a complete impermeability to sodium ions, or else that the cell actively extrudes them as fast as they enter. However, in view of what is already known about permeability to ions, it seems highly improbable that significant amounts of sodium will not penetrate into muscle fibres, if a period of, say, years is considered : yet in fact the ratio of sodium to potassium remains unchanged for years.

We must therefore regard it as probable that the sodium ion is actively extruded by muscle, and probably by many other cell types.

Considered as a whole, this discussion revealed several trends. First, it is apparent that the examination of the chemistry of biologically important molecules has reached a point at which it is possible, desirable, and necessary to obtain more information about cytochemical distributions: the biochemist can now begin to piece together the chemical fragments of cells with which he is familiar, and develop an understanding of how they are organised in the interests of the processes of life. Secondly, the general picture of the globular protein molecule now forming is one which is satisfactory to those using many different modes of study, and one which is acceptable to the cytologist. Thirdly, while in vitro studies of biologically important molecules can often profitably proceed quite independently of biophysics, when the biochemist turns from the analytical to the constructive sphere, the inexorable necessity for a concomitant physical approach becomes plain. For the study of the living cell, even at the simplest level of organisation, a marriage of true minds, the physical and the chemical, is essential. Let us admit no impediments to this. J. F. Danielli. 